\title{
Allele-specific marker-based assessment revealed that the rice blast resistance genes Pi2 and Pi9 have not been widely deployed in Chinese indica rice cultivars
}

Dagang Tian ${ }^{1,2}$, Zaijie Chen ${ }^{2}$, Ziqiang Chen ${ }^{2}$, Yuanchang Zhou ${ }^{{ }^{*}}$, Zonghua Wang ${ }^{3}$, Feng Wang ${ }^{2}$ and Songbiao Chen ${ }^{2^{*}}$

\begin{abstract}
Background: The most sustainable approach to control rice blast disease is to develop durably resistant cultivars. In molecular breeding for rice blast resistance, markers developed based on polymorphisms between functional and non-functional alleles of resistance genes, can provide precise and accurate selection of resistant genotypes without the need for difficult, laborious and time-consuming phenotyping. The Pi2 and Pi9 genes confer broad-spectrum resistance against diverse blast isolates. Development of allele-specific markers for Pi2 and Pi9 would facilitate breeding of blast resistant rice by using the two blast resistance genes.

Result: In this work, we developed two new markers, named Pi9-Pro and Pi2-LRR respectively, targeting the unique polymorphisms of the resistant and susceptible alleles of Pi2 and of Pi9. The InDel marker Pi9-Pro differentiates three different genotypes corresponding to the Pi2/Piz-t, Pi9 and non-Pi2/Piz-t/Pi9 alleles, and the CAPS marker Pi2-LRR differentiates the Pi2 allele from the non-Pi2 allele. Based on the two newly developed markers and two available markers Pi2SNP and PigSNP, the presence of Pi2 and Pi9 was assessed in a set of 434 rice accessions consisting of 377 Chinese indica cultivars/breeding materials and 57 Chinese japonica cultivars/breeding materials. Of the 434 accessions tested, while one indica restorer line Huazhan was identified harboring the Pi2 resistance allele, no other rice line was identified harboring the Pi2 or Pi9 resistance alleles.

Conclusions: Allele-specific marker-based assessment revealed that Pi2 and Pi9 have not been widely incorporated into diverse Chinese indica rice cultivars. Thus, the two blast resistance genes can be new gene sources for developing blast resistant rice, especially indica rice, in China. The two newly developed markers should be highly useful for using Pi2 and Pi9 in marker-assisted selection (MAS) breeding programs.
\end{abstract}

Keywords: Rice, Blast disease, Resistance gene, Molecular marker, Pi2, Pi9

\section{Background}

Rice blast, caused by the ascomycete fungus Magnaporthe oryzae, is the most destructive disease that affects rice production worldwide (Dean et al. 2005; Ebbole 2007). Deployment of resistant cultivars has been proved to be the most effective and environmentally-friendly

\footnotetext{
* Correspondence: zwy_2002@163.com; songbiao_chen@hotmail.com ${ }^{1}$ College of Crop Science, Fujian Agricultural and Forestry University, Fuzhou 350002, China

${ }^{2}$ Biotechnology Research Institute, Fujian Academy of Agricultural Sciences, Fuzhou 350003, China

Full list of author information is available at the end of the article
}

way to control rice blast disease (Zeigler et al. 1994). Numerous resistance $(R)$ genes conferring resistance to $M$. oryzae have been identified in diverse rice germplasm, and at least 23 blast $R$-genes have been cloned (Liu et al. 2014), providing a wealth of information and resources for improving blast resistance in rice.

Marker-assisted selection (MAS) has been employed to develop rice cultivars with resistance against blast disease. MAS allows rapid introgression of blast $R$-genes into susceptible varieties as well as pyramiding of multiple genes into a single line for more durable blast 
resistance. To date, a number of blast $R$-genes have been tagged by genetically linked DNA markers, such as SSR (simple sequence repeat) or other PCR-based markers. However, owing to genetic recombination, the genetically linked markers may give rise to false positives (Frisch et al. 1999). With the progress in cloning of blast $R$-genes and the availability of sequences of more functional $R$-genes, functional allele-specific markers have been developed for several blast $R$-genes, such as $P i-k m$ (Costanzo and Jia 2010), Pit (Hayashi et al. 2010), Pi54 (Ramkumar et al. 2011), Pi2/Piz-t/Pi9 (Hua et al. 2015), and Pita (Jia et al. 2002; Ramkumar et al. 2015) by using the strategy of allele mining. These allele-specific markers provide more efficient selection of desired genotypes compared to DNA markers at nearby, but functionally irrelevant site (Andersen and Lubberstedt 2003).

The Pi2/9 locus on the short arm of chromosome 6 contains at least nine identified blast $R$-genes, including Pi26(t) (Wu et al. 2005), Pigm(t) (Deng et al. 2006), Piz(t) (Fjellstrom et al. 2006), Pi9 (Qu et al. 2006), Pi2 (Zhou et al. 2006), Piz- $t$ (Zhou et al. 2006), Pi40(t) (Jeung et al. 2007), Pi2-2 (Jiang et al. 2012b), and Pi5O(t) (Zhu et al. 2012). Previous studies showed that many $R$-genes from the Pi2/9 locus confer broad-spectrum resistance to diverse M. oryzae isolates (Jiang et al. 2012b). The Pi2/9 locus, therefore, has been extensively used in MAS breeding program for improving blast resistance in rice (Chen et al. 2004; Hayashi et al. 2006; Liu et al. 2008; Suh et al. 2009; Correa-Victoria and Martinez 2009; Wen and Gao 2011; Yin et al. 2011; Fu et al. 2012; Jiang et al. 2012a; Luo and Yin 2013; Jiang et al. 2015; Khanna et al. 2015). Three R-genes (Pi2, Piz-t and Pi9) from the Pi2/9 locus have been cloned (Qu et al. 2006; Zhou et al. 2006). Sequence analyses revealed that Pi2, Piz-t and Pi9 encode nucleotide binding site-leucine-rich repeat (NBS-LRR) type resistance proteins, and the genes are embedded in a cluster of tandemly repeated NBS-LRR genes (Qu et al. 2006; Zhou et al. 2006). DNA markers linked to the Pi2 and Pi9 genes have been used frequently for MAS breeding of rice in China (Chen et al. 2004; Liu et al. 2008; Wen and Gao 2011; Yin et al. 2011; Fu et al. 2012; Jiang et al. 2012a; Jiang et al. 2015). Although genetically linked markers were suitable for screening, DNA markers targeting the polymorphisms that define the functional and non-functional alleles of $P i 2$ and of Pi9 would greatly enhance the efficiency and accuracy of genotype selection in MAS breeding programs. In the present study, we present the development of two allele-specific markers for Pi2 and Pi9. The markers were further used to assess the presence of the two blast $R$-genes in 434 rice accessions, consisting of 377 Chinese indica cultivars/breeding materials and 57 Chinese japonica cultivars/breeding materials. Among the 434 accessions, only the indica restorer line
Huazhan was identified harboring the Pi2 resistance allele, indicating that the two blast $R$-genes have not been widely incorporated into diverse Chinese indica rice cultivars. This suggests that Pi2 and Pi9 could be important new sources for developing blast resistant rice in China, especially in indica rice. The two newly developed markers should facilitate rapid incorporations of these resistance genes into rice through MAS breeding programs.

\section{Results}

Development of a marker targeting a conserved InDel in the promoter region of $P i 9$

Genomic DNA sequences corresponding to the Pi2/9 locus in cvs. C101A51 (Pi2-carrying isogenic line), 75-1127 (Pi9-carrying line), and Nipponbare were retrieved from Genbank and were used for comparative analysis. Because InDel (insertion-deletion) markers are more convenient for use in most regular laboratories compared with SNP (single nucleotide polymorphism) markers, we focused firstly on InDel polymorphisms among the sequences and found that the region corresponding to the promoter of $\mathrm{Pi} 9$ and its alleles contains InDel polymorphisms with potential as molecular markers (Fig. 1a). To further verify the identified InDel polymorphisms, PCR fragments corresponding to the promoter region of $P i 9$ and its alleles were amplified from C101A51, Toride-1 (Piz-t-carrying line), 75-1-127, Nipponbare, and 22 other cultivars (Fig. 1b) and were sequenced. Through multiple sequence alignment, a 10bp InDel variation positioned at $-516 /-517 \mathrm{bp}$ in the promoter region of $P i 9$ in 75-1-127 (relative to the ATG start codon of $P i 9$ ) was identified that was not present in any of the other 25 cultivars (Fig. 1a-b). Interestingly, a 27-bp InDel close to the 10-bp InDel was also identified in sequences from C101A51 and Toride-1 that was not found in the other 24 cultivars (Fig. 1b). This 27-bp InDel is located about $28.3 \mathrm{~kb}$ upstream of the coding region of Pi2/Piz-t (Fig. 1a). Based on the sequences alignment result, a pair of specific primers, 9-Pro-F/9Pro-R (Additional file 1: Table S1), targeted to the region containing the identified InDels was designed to develop molecular marker. Using the primers 9-Pro-F/9-Pro-R, PCR analysis detected an expected 111-bp fragment in both C101A51 and Toride-1, and an expected 128-bp fragment in 75-1-127 (Fig. 1c). In contrast, PCR analysis detected a 138-bp fragment in all other cultivars (Fig. 1c). Therefore, the InDel marker (named Pi9-Pro) can differentiate three different genotypes corresponding to the Pi2/Piz-t, Pi9 and non-Pi2/Piz-t/Pi9 alleles.

To validate the linkage of the Pi9-Pro marker with the Pi2- and Pi9-resistance phenotype, two $\mathrm{F}_{2}$ populations from the crosses of $\mathrm{C} 101 \mathrm{~A} 51 \times \mathrm{CO} 39$ and 75-1-127 $\times$ CO39 respectively, were inoculated with the $M$. oryzae 


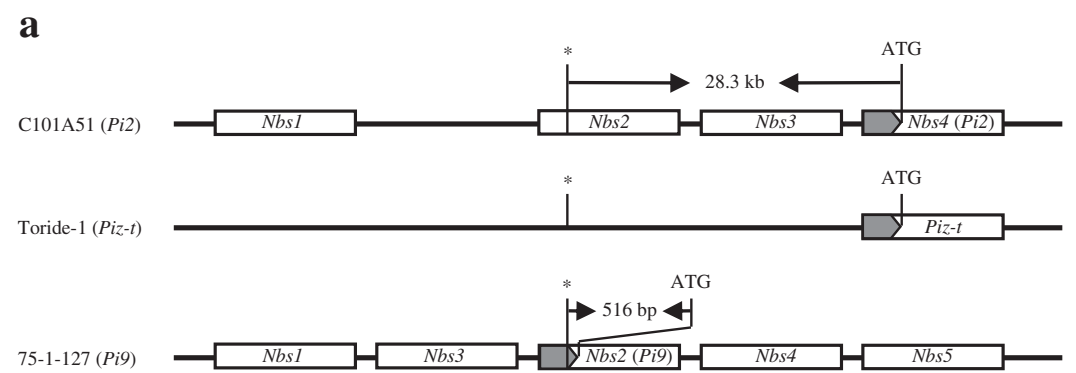

b

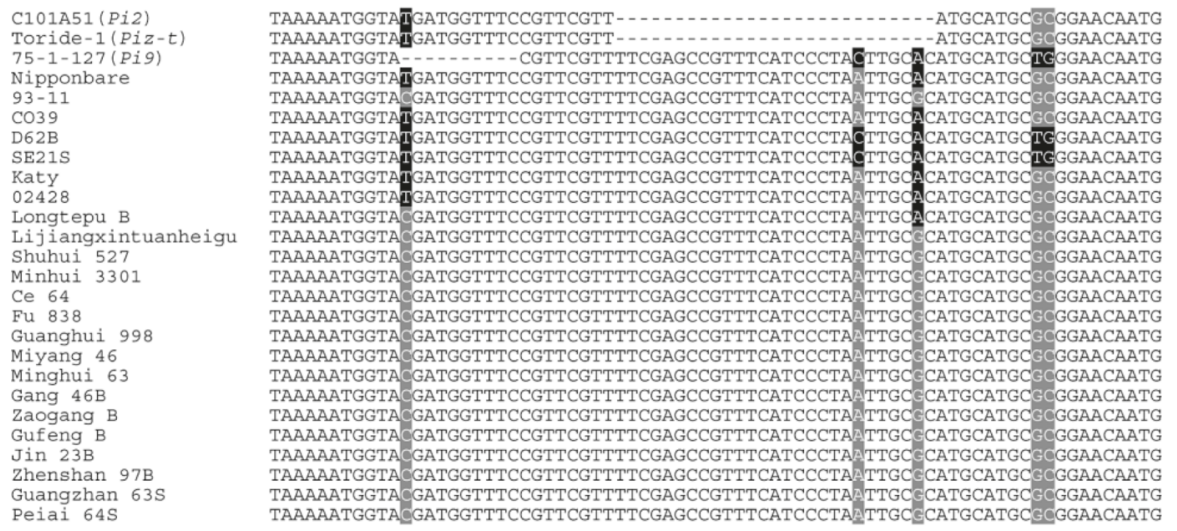

Peiai $64 \mathrm{~S}$ TAAAAATGGAGGATGGTTCCGTTCGTTTTCGAGCCGTTTCATCCCTAMITTCGCATGCATGCGCGGAACAATG

C

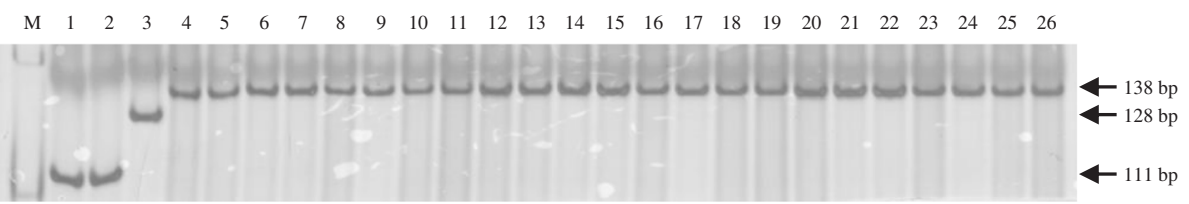

Fig. 1 An InDel marker targeting the promoter region of Pi9. a Physical location of the identified InDel region in the Pi2/9 locus. b Sequence alignment showing the 27-bp InDel between the Pi2/Piz- $t$ and non-Pi2/Piz- $t$ alleles and the 10-bp InDel between the Pi9 and non-Pi9 alleles in diverse cultivars. c PCR amplification patterns of the InDel marker Pi9-Pro that differentiates the Pi2/Piz-t, Pi9 and non-Pi2/Piz-t/Pi9 alleles. M: DNA ladder; 1: C101A51 (Pi2 donor line); 2: Toride-1 (Piz-t donor line); 3: 75-1-127 (Pi9 donor line); 4: Nipponbare; 5: 93-11; 6: CO39; 7: D62B; 8: SE21S; 9: Katy; 10: 02428; 11: Longtepu B; 12: Lijiangxintuanheigu; 13: Shuhui 527; 14: Minhui 3301; 15: Ce 64; 16: Fu 838; 17: Guanghui 998; 18: Miyang 46; 19: Minghui63; 20: Gang 46B; 21: Zaogang B; 22: Gufeng B; 23: Jin 23B; 24: Zhenshan 97B; 25: Guangzhan 63S; 26: Peiai 64S. Asterisk indicates the InDel region; Grey arrows indicate the promoter regions of Pi2, Piz-t or Pi9

isolate PO6-6 which was incompatible to both $\mathrm{Pi2}$ and $P i 9$. In the $\mathrm{F}_{2}$ segregating population of C101A51 × CO39, PCR analysis based on primers 9-Pro-F/9-Pro-R detected a 111-bp fragment in either homozygous or heterozygous patterns in all 134 resistant plants. By contrast, PCR amplification yielded only a 138-bp fragment in all 40 susceptible plants. Similarly, the 128-bp genotype of the Pi9-Pro marker corresponding to Pi9 allele was found to cosegregate with the blast-resistance phenotype in the $F_{2}$ segregating population of 75-1-157 × CO39.

\section{Development of a CAPS marker targeting the LRR domain of Pi2}

The Pi9-Pro marker was unable to differentiate between the Pi2 and Piz-t alleles. To further develop a marker that can be used to discriminate Pi2 from Piz-t or from other non-Pi2 alleles, we focused on the polymorphic sites within the coding regions of Pi2 and Piz-t. As previously reported, Pi2 and Piz-t displayed eight aminoacid differences within three leucine-rich repeat (LRR) motifs (Fig. 2a) (Zhou et al. 2006). Alignment of DNA sequences of Pi2 and its alleles from C101A51, Toride-1, 75-1-127, and Nipponbare revealed that the polymorphic variations corresponding to the first six amino-acid differences (AA788, AA789, AA790, AA792, AA-/793, and AA793/794; Fig. 2a) were conserved among different alleles (Fig. 2b). To further verify the polymorphisms for marker development, PCR fragments corresponding to the identified polymorphic region were amplified from C101A51, Toride-1, 75-1-127, Nipponbare and 20 other cultivars (Fig. 2b), and were sequenced. Multiple sequence alignment of the PCR products revealed that 
a

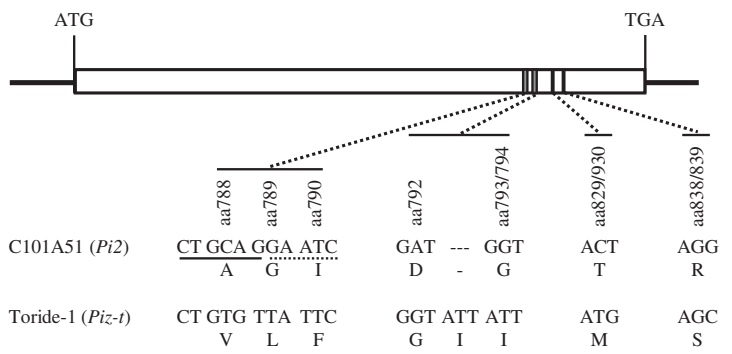

b

C101A51 (Pi2) Toride-1 (Piz-t) 75-1-127 (Pig) Nipponbare 93-11 $\mathrm{CO} 39$ D62B Xianghui 68 Shuhui 527 Zhenshan 978 Minghui 63 Guangzhan $63 \mathrm{~S}$ Gang $46 \mathrm{~B}$ Miyang 46 $\mathrm{Fu} 838$ SE21S Peiai 64S Lijiangxint Longtepu B 02428 Guanghui 998 $\mathrm{Jin} 23 \mathrm{~B}$
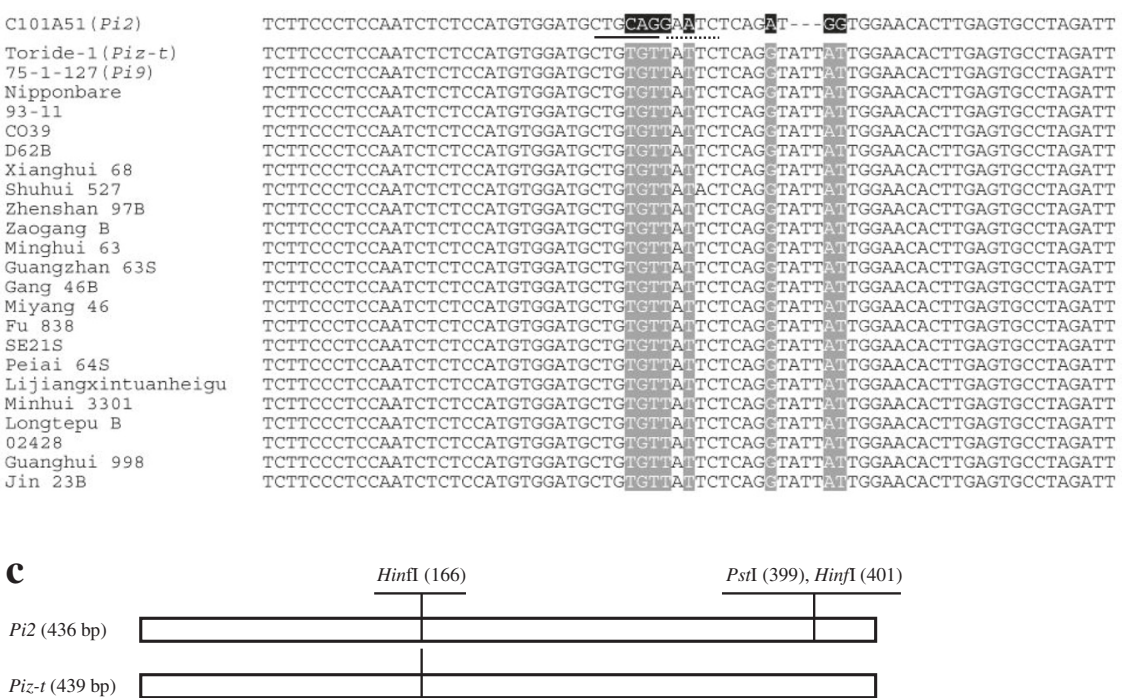

d

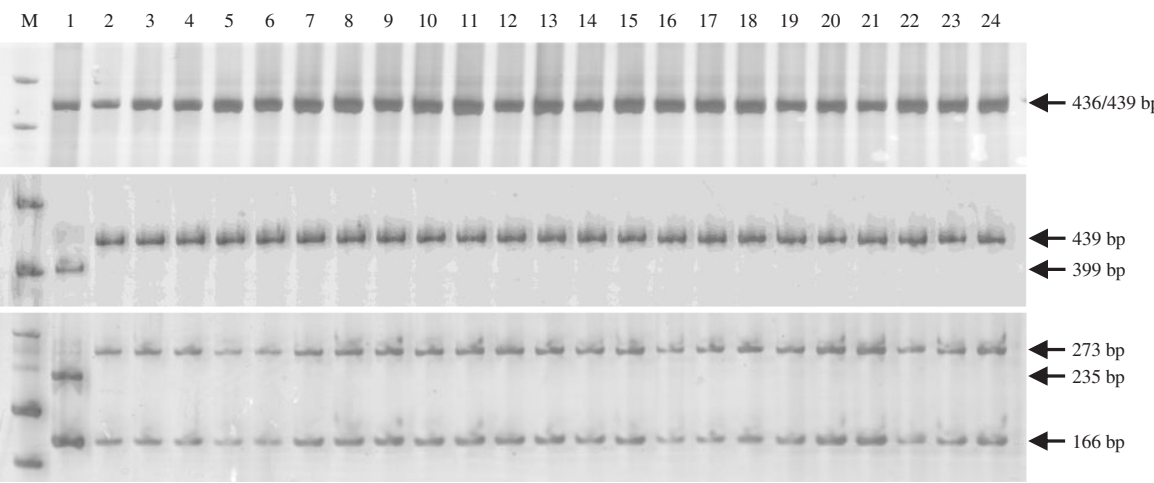

Fig. 2 A CAPS marker targeting the LRR domain of Pi2. a DNA polymorphisms corresponding to the eight amino-acid differences in between Pi2 and Piz-t. b Sequence alignment showing that the polymorphic variations corresponding to the first six amino-acid differences in between Pi2 and Piz-t were conserved between the Pi2 and non-Pi2 alleles in diverse cultivars. c Schematic diagrams indicating sizes and recognition sites for Hinfl and Pstl in the PCR fragment of the Pi2 and Piz-t alleles amplified with primers 2-LRR-F/2-LRR-R. $\mathbf{d}$ Electrophoresis profiles of the CAPS marker Pi2-LRR that differentiates the Pi2 and non-Pi2 alleles. Upper panel showing the PCR products without digestion with restriction enzymes, middle panel showing the PCR products digested with Pstl, and lower panel showing the PCR products digested with Hinfl. M: DNA ladder; 1: C101A51 (Pi2 donor line); 2: Toride-1 (Piz-t donor line); 3: 75-1127 (Pi9 donor line); 4: Nipponbare; 5: 93-11; 6: CO39; 7: D62B; 8: Xianghui 68; 9: Shuhui 527; 10: Zhenshan 97B; 11: Zaogang B; 12: Minghui 63; 13: Guangzhan 63S; 14: Gang 46B; 15: Miyang 46; 16: Fu 838; 17: SE21S; 18: Peiai 64S; 19: Lijiangxintuanheigu; 20: Minhui 3301; 21: Longtepu B; 22: 02428; 23: Guanghui 998; 24: Jin 23B. Pstl recognition sequence (CTGCAG) is underlined; Hinfl recognition sequence (GAATC) is marked by dotted underling

the polymorphic variations were conserved between Pi2 and alleles of the other 23 tested cultivars (Fig. 2b). Sequence analysis also revealed that the variations defining the functional Pi2 gene formed two recognition sites located closely together for restriction endonucleases Pst I and HinfI, respectively (Fig. 2c). 
Based on the sequence variations, a CAPS (cleaved amplified polymorphic sequence) marker (named Pi2LRR) was designed for Pi2. Using a pair of specific primers, 2-LRR-F/2-LRR-R (Additional file 1: Table S1), PCR amplification yielded fragments of $436 \mathrm{bp}$, or 439 bp for Pi2 and Piz- $t$ alleles, respectively (Fig. 2c-d). Upon digestion with PstI or HinfI, the PCR products showed discernible patterns between the Pi2 and Piz-t alleles (Fig. 2c-d). For example, following digestion with PstI, the Pi2-PCR product was reduced to a $399 \mathrm{bp}$ fragment, and the Piz-t-PCR product remained intact with its original size of $439 \mathrm{bp}$. Using the 2-LRR-F/2-LRR-R primers, PCR fragments were successfully amplified from genomic DNAs of C101A51, Toride-1, 75-1-127, Nipponbare and 20 other cultivars. Digestion analysis of the PCR products showed that the Pi2-LRR marker could differentiate the Pi2 allele from the non-Pi2 allele in diverse rice cultivars (Fig. 2d). The Pi2-LRR marker was also validated in a $F_{2}$ segregating population derived from the cross of C101A $51 \times \mathrm{CO} 39$, and the result showed that the genotype corresponding to Pi2 allele for the Pi2-LRR marker co-segregated with the blastresistance phenotype.

\section{Assessment of the Pi2 and Pi9 genes in diverse Chinese indica rice cultivars}

The Pi9-Pro and Pi2-LRR markers were further validated for their specificity to $\mathrm{Pi} 2$ or $\mathrm{Pi} 9$ in monogenic lines harboring blast resistance genes in the Pi2/9 locus, including IRBLz5-CA (Piz-5 = Pi2), IRBL9-W (Pi9), IRBLzt-T $(P i z-t)$ and IRBLz-Fu (Piz) (Tsunematsu et al. 2000).
PCR analysis based on primers 9-Pro-F/9-Pro-R detected a Pi9-type genotype and a Pi2/Piz-t-type genotype in IRBL9-W and in IRBLz5-CA/IRBLzt-T (Table 2), respectively. As for the Pi2-LRR marker, PCR analysis detected a Pi2-type genotype only in IRBLz5-CA (Table 2).

Besides the donor and monogenic lines, a set of 434 Chinese rice cultivars or breeding materials used in China were assessed for the presence of the Pi2 and Pi9 genes. The 434 rice accessions consisted of 91 indica restorer lines of hybrid rice, 61 indica maintainer/sterile lines of hybrid rice, 225 indica conventional cultivars/ breeding materials, and 57 japonica conventional cultivars/breeding materials (Additional file 2: Table S2). When using the Pi9-Pro marker for screening, 15 out of 434 accessions were identified displaying a 111-bp PCR fragment (Table 1, Table 2, Fig. 3), suggesting that these 15 accessions might contain the Pi2 or Piz-t resistance genes. In contrast, no accessions were detected harboring a 128-bp PCR fragment corresponding to the Pi9 allele (Table 1), suggesting that the tested accessions did not have the Pi9 resistance gene. When using the Pi2LRR marker for screening, no tested accession except an indica restorer line Huazhan produced digestion patterns identical to that of the Pi2 allele (Table 1, Table 2, Fig. 3). Notably, Huazhan was among the 15 accessions that displayed a 111-bp PCR fragment corresponding to the Pi2/Piz- $t$ alleles as identified by the Pi9-Pro marker. Taken together, these results suggested that Huazhan might contain the Pi2 resistance gene.

The 434 rice accessions were also screened with two CAPS/dCAPS (derived cleaved amplified polymorphic

Table 1 Summary of genotyping results of the four molecular markers in 434 Chinese rice cultivars or breeding materials

\begin{tabular}{|c|c|c|c|c|c|c|c|c|}
\hline \multirow[t]{3}{*}{ Marker } & \multirow{3}{*}{$\begin{array}{l}\text { Genotype } \\
\text { (bp) }\end{array}$} & \multirow{3}{*}{$\begin{array}{l}\text { Corresponding } \\
\text { donor line }\end{array}$} & \multicolumn{4}{|c|}{ No. of cultivars or breeding materials } & \multirow{3}{*}{$\begin{array}{l}\text { Japonica } \\
\text { Conventional cultivars } \\
\text { or breeding materials }\end{array}$} & \multirow[t]{3}{*}{ Total } \\
\hline & & & & \multicolumn{3}{|l|}{ Indica } & & \\
\hline & & & & $\begin{array}{l}\text { Restorer lines } \\
\text { of hybrid rice }\end{array}$ & $\begin{array}{l}\text { Maintainer or sterile } \\
\text { lines of hybrid rice }\end{array}$ & $\begin{array}{l}\text { Conventional cultivars } \\
\text { or breeding materials }\end{array}$ & & \\
\hline & & & Total & 91 & 61 & 225 & 57 & 434 \\
\hline \multirow[t]{3}{*}{ Pig-Pro } & 138 & & & 86 & 61 & 220 & 52 & 419 \\
\hline & 128 & 75-1-127 (Pi9) & & 0 & 0 & 0 & 0 & 0 \\
\hline & 111 & $\begin{array}{l}\text { C101A51 (Pi2) } \\
\text { Toride-1 (Piz-t) }\end{array}$ & & 5 & 0 & 5 & 5 & 15 \\
\hline \multirow[t]{4}{*}{ Pi2-LRR } & Pstl-439 & & & 90 & 61 & 225 & 57 & 433 \\
\hline & Pstl-399 & C101A51 (Pi2) & & 1 & 0 & 0 & 0 & 1 \\
\hline & Hinfl-273 & & & 90 & 61 & 225 & 57 & 433 \\
\hline & Hinfl-235 & C101A51 (Pi2) & & 1 & 0 & 0 & 0 & 1 \\
\hline \multirow[t]{2}{*}{ Pi2SNPa } & 267 & & & 90 & 61 & 225 & 57 & 433 \\
\hline & 235 & C101A51 (Pi2) & & 1 & 0 & 0 & 0 & 1 \\
\hline \multirow[t]{2}{*}{ PigSNP } & 126 & & & 91 & 61 & 225 & 57 & 434 \\
\hline & 108 & 75-1-127 (Pi9) & & 0 & 0 & 0 & 0 & 0 \\
\hline
\end{tabular}

${ }^{\text {a }}$ i2-type genotype for Pi2SNP is $235 \mathrm{bp}$

${ }^{\mathrm{b}}$ Pig-type genotype for Pi9SNP is 108 bp 
Table 2 List of the 15 cultivars displaying a Pi2/Piz-t-type genotype for the Pi9-Pro marker

\begin{tabular}{|c|c|c|c|c|c|c|c|c|c|c|c|c|c|c|c|c|c|}
\hline \multirow[t]{3}{*}{ Subspecies } & \multirow[t]{3}{*}{ Category } & \multirow[t]{3}{*}{ Cultivars } & \multicolumn{5}{|c|}{ Molecular marker genotype (bp) } & \multicolumn{10}{|c|}{ Disease reactions } \\
\hline & & & \multirow[t]{2}{*}{ Pi9-Pro } & \multicolumn{2}{|c|}{ Pi2-LRR } & \multirow[t]{2}{*}{ Pi2SNPa } & \multirow[t]{2}{*}{ PigSNPb } & \multirow[t]{2}{*}{ KJ201 } & \multirow[t]{2}{*}{ CHE86 } & \multirow[t]{2}{*}{ CHNOS } & \multirow[t]{2}{*}{ RB16 } & \multirow[t]{2}{*}{ RB22 } & \multirow[t]{2}{*}{ HYG99 } & \multirow[t]{2}{*}{ IR16 } & \multirow[t]{2}{*}{ JL311 } & \multirow[t]{2}{*}{ JL501 } & \multirow[t]{2}{*}{ ZY838 } \\
\hline & & & & Pstl & Hinfl & & & & & & & & & & & & \\
\hline & Reference line & Nipponbare & 138 & 439 & 273 & 267 & 126 & S & $S$ & S & S & $S$ & $S$ & $S$ & S & S & $S$ \\
\hline & Donor lines & C101A51 (Pi2) & 111 & 399 & 235 & 235 & 126 & $\mathrm{R}$ & $\mathrm{R}$ & s & $\mathrm{R}$ & S & R & R & $\mathrm{R}$ & $\mathrm{R}$ & $\mathrm{R}$ \\
\hline & & Toride-1 (Piz-t) & 111 & 439 & 273 & 267 & 126 & $\mathrm{R}$ & R & $\mathrm{R}$ & S & s & S & S & s & S & S \\
\hline & & 75-1-127 (Pi9) & 128 & 439 & 273 & 267 & 108 & $\mathrm{R}$ & $R$ & $\mathrm{R}$ & R & $\mathrm{R}$ & $\mathrm{R}$ & R & $\mathrm{R}$ & $\mathrm{R}$ & $\mathrm{R}$ \\
\hline & Monogenic lines & IRBLz5-CA (Pi2) & 111 & 399 & 235 & 235 & 126 & ND & ND & ND & ND & ND & ND & ND & ND & ND & ND \\
\hline & & IRBLzt-T (Piz-t) & 111 & 439 & 273 & 267 & 126 & ND & ND & ND & ND & ND & ND & ND & ND & ND & ND \\
\hline & & IRBL9-W (Pi9) & 128 & 439 & 273 & 267 & 108 & ND & ND & ND & ND & ND & ND & ND & ND & ND & ND \\
\hline & & IRBLz-FU (Piz) & 138 & 439 & 273 & 267 & 126 & ND & ND & ND & ND & ND & ND & ND & ND & ND & ND \\
\hline \multirow[t]{10}{*}{ Indica } & \multirow[t]{5}{*}{ Restorer lines of hybrid rice } & Duohui 43 & 111 & 439 & 273 & 267 & 126 & $\mathrm{R}$ & R & $\mathrm{R}$ & $\mathrm{R}$ & $\mathrm{R}$ & $\mathrm{R}$ & R & S & s & $S$ \\
\hline & & Minghui 86 & 111 & 439 & 273 & 267 & 126 & $\mathrm{R}$ & R & $\mathrm{R}$ & $\mathrm{R}$ & S & $\mathrm{R}$ & R & S & R & $\mathrm{R}$ \\
\hline & & Huazhan & 111 & 399 & 235 & 235 & 126 & $\mathrm{R}$ & $\mathrm{R}$ & $\mathrm{R}$ & $\mathrm{R}$ & $\mathrm{R}$ & $\mathrm{R}$ & $\mathrm{R}$ & $\mathrm{R}$ & $\mathrm{R}$ & $\mathrm{R}$ \\
\hline & & Minghui 1259 & 111 & 439 & 273 & 267 & 126 & $\mathrm{R}$ & $\mathrm{R}$ & $\mathrm{R}$ & $\mathrm{R}$ & S & $\mathrm{R}$ & S & S & S & $\mathrm{R}$ \\
\hline & & Xianghui 68 & 111 & 439 & 273 & 267 & 126 & $\mathrm{R}$ & $\mathrm{R}$ & $\mathrm{R}$ & S & S & S & R & S & S & S \\
\hline & \multirow{5}{*}{$\begin{array}{l}\text { Conventional cultivars and } \\
\text { breeding materials }\end{array}$} & Fengxinzhan & 111 & 439 & 273 & 267 & 126 & $\mathrm{R}$ & R & $\mathrm{R}$ & $\mathrm{R}$ & $\mathrm{R}$ & S & R & S & S & $\mathrm{R}$ \\
\hline & & GIZA 176 & 111 & 439 & 273 & 267 & 126 & $\mathrm{R}$ & R & R & $\mathrm{R}$ & S & S & R & $\mathrm{R}$ & R & R \\
\hline & & Jiabala & 111 & 439 & 273 & 267 & 126 & $\mathrm{R}$ & R & R & $\mathrm{R}$ & $\mathrm{R}$ & S & S & $\mathrm{R}$ & R & R \\
\hline & & Jiazao No.1 & 111 & 439 & 273 & 267 & 126 & R & R & $\mathrm{R}$ & R & $\mathrm{R}$ & R & R & S & R & R \\
\hline & & Jiazao 935 & 111 & 439 & 273 & 267 & 126 & $\mathrm{R}$ & $\mathrm{R}$ & $\mathrm{R}$ & S & S & S & S & S & S & $\mathrm{R}$ \\
\hline \multirow[t]{5}{*}{ Japonica } & \multirow{5}{*}{$\begin{array}{l}\text { Conventional cultivars and } \\
\text { breeding materials }\end{array}$} & Haomake (K) & 111 & 439 & 273 & 267 & 126 & R & R & $\mathrm{R}$ & R & S & R & R & S & S & R \\
\hline & & Lemont & 111 & 439 & 273 & 267 & 126 & $\mathrm{R}$ & $\mathrm{R}$ & $\mathrm{R}$ & $\mathrm{R}$ & $\mathrm{R}$ & $\mathrm{R}$ & $\mathrm{R}$ & S & R & $\mathrm{R}$ \\
\hline & & Shuiyuan 290 & 111 & 439 & 273 & 267 & 126 & $\mathrm{R}$ & $\mathrm{R}$ & R & $\mathrm{R}$ & S & R & R & $\mathrm{R}$ & S & $\mathrm{R}$ \\
\hline & & Shuiyuan 377 & 111 & 439 & 273 & 267 & 126 & R & $\mathrm{R}$ & $\mathrm{R}$ & R & R & S & S & S & $S$ & S \\
\hline & & Zhonghua No.8 & 111 & 439 & 273 & 267 & 126 & $\mathrm{R}$ & $\mathrm{R}$ & $\mathrm{R}$ & $\mathrm{R}$ & $\mathrm{R}$ & S & S & S & $\mathrm{R}$ & S \\
\hline
\end{tabular}

$S$ susceptible, $R$ resistant, $N D$ not determined

a Pi2-type genotype for Pi2SNP is 235 bp

${ }^{\text {b }}$ Pig-type genotype for Pi9SNP is 108 bp 


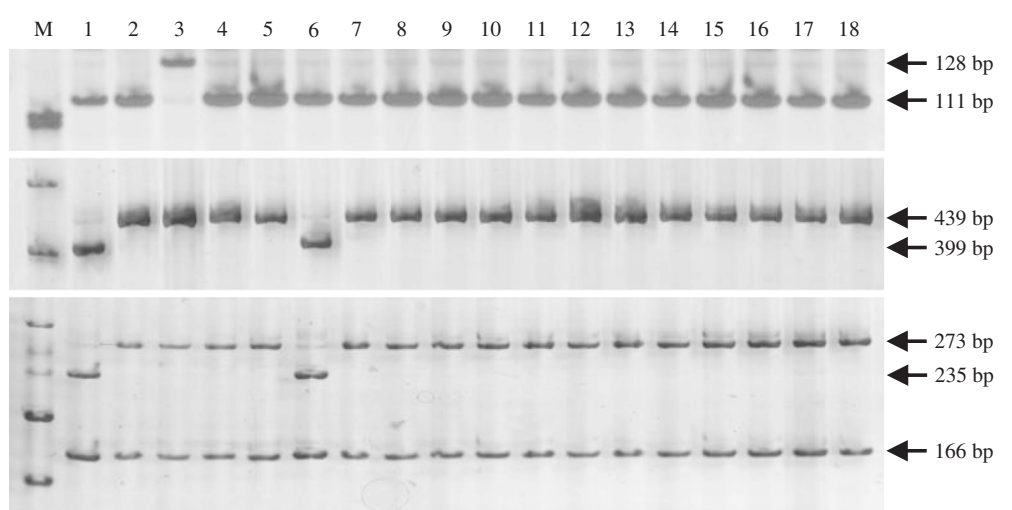

Fig. 3 Pi9-Pro and Pi2-LRR analyses of 15 cultivars displaying a Pi2/Piz-t-type genotype for the Pi9-Pro marker. Upper panel showing the PCR amplification patterns of the InDel marker Pi9-Pro; Middle panel showing the patterns of Pi2-LRR products digested with Pstl; and lower panel showing the patterns of Pi2-LRR products digested with Hinfl. M: DNA ladder; 1: C101A51 (Pi2 donor line); 2: Toride-1 (Piz-t donor line); 3: 75-1-127 (Pi9 donor line); 4: Duohui 43; 5: Minghui 86; 6: Huazhan; 7: Minghui 1259; 8: Xianghui 68; 9: Fengxinzhan; 10: GIZA 176; 11: Jiabala; 12: Jiazao No.1; 13: Jiazao 935; 14: Haomake (K); 15: Lemont; 16: Shuiyuan 290; 17: Shuiyuan 377; 18: Zhonghua No.8

sequence) markers Pi2SNP and Pi9SNP which were developed based on SNPs within the coding region of Pi2 and $P i 9$, respectively (Hua et al. 2015). The genotyping results of the Pi2SNP and Pi9SNP markers were consistent with that of the Pi2-LRR and Pi9-Pro markers. Pi2SNP analysis identified only one (cv. Huazhan) out of 434 accessions giving a restriction enzyme digestion pattern identical to that of the Pi2 allele (Table 1, Table 2), and no accession was identified having a Pi9SNP genotype corresponding to the Pi9 allele (Table 1).

\section{Disease reaction of cultivars possessing a Pi2/Piz-t-type genotype for the Pi9-Pro marker}

Fifteen accessions were identified having a Pi9-Pro genotype identical to that of Pi2/Piz- $t$ alleles (Table 2). Further genotyping of the 15 cultivars using Pi2-LRR and Pi2SNP markers strongly suggested that Huazhan harbors the Pi2 resistance gene. To investigate whether the Pi2/Piz-t-type cultivars showed Pi2- or Piz-t-specific resistance, the 15 cultivars were inoculated with two blast isolates KJ201 and CHE86, which were incompatible to Pi2, Piz-t and Pi9; one isolate, CHNOS, which was compatible to Pi2, but incompatible to Piz-t and Pi9 (Zhou et al. 2006); and seven isolates collected from Fujian province, China, which were compatible to Piz-t. As shown in Table 2, the Pi2-type cultivar Huazhan appeared resistant to isolates KJ201, CHE86 and CHNOS, consistent with the disease reactions in the Pi2-carrying isogenic line C101A51, except that C101A51 was susceptible to CHNOS. The other 14 Piz-t-type cultivars showed resistance to KJ201, CHE86 and CHNOS, consistent with the disease reactions in the Piz-t-carrying line Toride-1. As for inoculations with the seven Fujian isolates, the 15 cultivars showed differences in resistance spectrum compared with the Pi2 or Piz-t donor lines, suggesting that Huazhan and the 14 Piz-t-type cultivars probably possess other blast $R$-genes conferring resistance to CHNOS or the Fujian isolates.

\section{Discussion}

The use of resistant cultivars is an effective approach to control crop diseases. Over the past many decades, conventional breeding has had great success in improving rice resistance to blast disease. However, the traditional phenotype-based approach for breeding resistant cultivars is extremely laborious and time-consuming, and is often hampered by difficulty in screening the desired resistant progeny under field or limited inoculation conditions. In recent years, MAS breeding programs are being applied as an important supplement to the conventional phenotypic selection. Based on DNA molecular markers linked to resistance genes, MAS permits the direct selection of genes that control the disease resistance phenotype, providing an alternative to overcome the limitations of conventional breeding (Jena and Mackill 2008).

The efficiency of MAS mainly depends on the convenience of the use of DNA markers and the degree of linkage of the markers with the desired traits. Three major types of DNA markers, SSR, InDel and SNP markers have been frequently used in rice research and molecular breeding. Because of their co-dominance, capacity of multi-allelic detection and ease of use, SSR markers have been widely used for genotyping and MAS programs in rice (McCouch et al. 2002). For example, many blast $R$-genes were mapped based on SSR markers (Ashkani et al. 2016), and these markers linked to the blast $R$-genes have been thus applied in breeding of blast-resistant rice. However, due to the relatively low density of SSR markers, most of these SSR markers did not completely co-segregate with the mapped blast $R$ - 
genes, which can lead to false positive selections. SNP and InDel polymorphisms are very abundant in the rice genome, providing potential targets for designing allele-specific markers directly targeting distinct blast- $R$ gene alleles (Costanzo and Jia 2010; Hayashi et al. 2010; Ramkumar et al. 2011; Hua et al. 2015; Ramkumar et al. 2015). Because of their co-segregation with target blast $R$-genes (Ramkumar et al. 2011; Ramkumar et al. 2015), allelespecific markers are more effective than the random genomic SSR marker, allowing for selection of target blast $R$ genes with less labor and cost in MAS programs.

The Pi2 and Pi9 genes confer broad-spectrum resistance against diverse $M$. oryzae isolates (Liu et al. 2002; Qu et al. 2006; Zhou et al. 2006). Although several DNA markers have been developed for Pi2 (Chen et al. 2004; Liu et al. 2008; Fu et al. 2012; Jiang et al. 2012a; Jiang et al. 2015; Khanna et al. 2015; Hua et al. 2015) and Pi9 (Wen and Gao 2011; Yin et al. 2011; Luo and Yin 2013; Khanna et al. 2015; Hua et al. 2015), many of the markers were designed based on SSR polymorphisms or structural variants located some distance from the Pi2 or Pi9 genes. More recently, two CAPS/dCAPS markers targeting SNPs in the coding regions of Pi2 and Pi9 were reported (Hua et al. 2015). However, these two markers have not been validated for blast resistance. In the present study, we developed two new markers, Pi9-Pro and Pi2-LRR, targeting the unique polymorphisms in between the resistant and susceptible alleles of the two blast-R genes, respectively. The newly developed Pi9-Pro marker differentiates three different genotypes corresponding to the Pi2/Piz-t, Pi9, and nonPi2/Piz-t/Pi9 alleles. The Pi9-Pro marker was validated in $F_{2}$ segregating populations, and the results confirmed that both the Pi2-type and Pi9-type genotypes for Pi9-Pro perfectly co-segregated with the blast resistance phenotype. The Pi9-Pro marker was developed based on InDel polymorphisms. Unlike CAPS/dCAPS markers (Hua et al. 2015) which require digestion of PCR amplicons for genotyping, the newly developed Pi9-Pro InDel marker eliminates this extra laborious step, and reduces potential sources of error (Liu et al. 2015). Thus, the Pi9-Pro marker is advantageous for MAS breeding programs targeting these resistance genes, especially for large-scale population screening. The Pi9-Pro marker, however, was unable to differentiate between the Pi2 and Piz- $t$ alleles. Pi2-LRR is a CAPS marker targeting the LRR motifs of the coding region of Pi2. The Pi2-LRR marker differentiates the Pi2 allele from the Piz- $t$ allele, and from other non-Pi2 alleles. Pi2-LRR was also validated in a $F_{2}$ segregating population, which showed that the Pi2-type genotype of the marker cosegregated with the blast resistance phenotype. Thus, when Pi9-Pro serves as a base marker for large-scale screening of Pi2 in MAS breeding programs, Pi2-LRR can be used as a supplementary marker for confirmative screening, enabling more precise selection of $\mathrm{Pi} 2$ in rice breeding populations.
To further assess the potential value of the $\mathrm{Pi2}$ and $\mathrm{Pi} 9$ genes in breeding of blast resistant rice in China, a set of 434 Chinese rice cultivars or breeding materials, mainly consisted of indica cultivars, were screened with the Pi9Pro and Pi2-LRR markers as well as the previously developed markers Pi2SNP and Pi9SNP (Hua et al. 2015). The screening revealed only one out of 434 rice accessions possessed the Pi2 resistance allele, and no cultivar possessed the $P i 9$ resistance allele, indicating that both the $P i 2$ and $P i 9$ genes have not been widely deployed in Chinese indica rice cultivars. Since only 57 japonica cultivars were screened in the present study, whether the $P i 2$ and $P i 9$ genes have been widely incorporated into Chinese japonica rice cultivars remains to be further investigated. The indica restorer line Huazhan, which was identified possessing the Pi2 allele, was bred from a Malaysian rice cultivar SC02-S6 (China Rice Data Center, http://www.ricedata.cn/variety/ varis/607962.htm?607962). Thus, the Pi2 allele in Huazhan was possibly derived from SC02-S6. In addition to Huazhan, 14 additional accessions displayed a Pi2/Piz-t-type genotype when screened with the Pi9-Pro marker. Further screening with the Pi2-LRR, Pi2SNP markers revealed that none of the 14 lines possesses the Pi2 allele, suggesting that these lines possibly carry the Piz-t allele. The 14 Piz-t-type accessions included four indica restorer lines, five indica conventional cultivars/breeding materials, and five japonica conventional cultivars/breeding materials (Table 2). Thus, the Piz-t gene appears to have been incorporated into diverse Chinese rice cultivars. The inoculation experiments showed that the 15 Pi2/Piz-t-type cultivars were resistant to isolates incompatible to Pi2 or Piz-t. When inoculated with isolates compatible to Pi2 or Piz-t, the Pi2/Piz-t-type cultivars showed resistance to some isolates. This could be explained by the cultivars possessing other blast $R$-genes conferring resistance to some of the isolates. The inoculation results also showed that compared with the Piz-t donor line Toride-1, the Pi2 donor line C101A51 and the $P i 9$ donor line 75-1-127 were resistant to more Fujian isolates (Table 2), suggesting that $\mathrm{Pi} 2$ and $\mathrm{Pi} 9$ have more potential value in improving blast resistance in the Fujian area.

\section{Conclusion}

In this study, we developed two allele-specific markers for Pi2 and Pi9, and evaluated the presence of the two blast $R$-genes in diverse Chinese cultivars/breeding materials based on allele-specific marker analysis. The assessment revealed that $P i 2$ and $P i 9$ have not been widely incorporated into diverse Chinese indica rice cultivars, supporting that the two blast- $R$ genes can be novel gene sources for breeding of blast resistant indica rice in China. Furthermore, the newly developed markers will be highly useful in tracking Pi2 and Pi9 for blast resistance in breeding programs. 


\section{Methods}

\section{Plant materials and blast isolates}

Blast R-gene donors C101A51 (carrying Pi2), Toride-1 (carrying Piz-t) and 75-1-127 (carrying Pi9) were kindly provided by Dr. Guo-Liang Wang (Department of Plant Pathology, Ohio State University). The 434 rice cultivars and breeding materials (Additional file 2: Table S2) were maintained at the Fujian Provincial Key Laboratory of Genetic Engineering for Agriculture, Fujian Academy of Agricultural Sciences, Fuzhou, China. $F_{2}$ populations derived from crosses between C101A51 and CO39, and between 75-1-127 and CO39 were used to validate the developed markers. The M. oryzae isolates PO6-6, KJ201, CHE86, and CHNOS were kindly provided by Dr. GuoLiang Wang (Department of Plant Pathology, Ohio State University). Seven M. oryzae isolates RB16, RB22, HYG99, IR16, JL311, JL501, and ZY838 collected from Fujian province, China, were also used in the study.

\section{Rice blast inoculations}

Rice blast inoculations were performed in a greenhouse with a fogging system to maintain a high level of humidity. Rice seedlings were grown in the greenhouse in cement tanks filled with puddled soil for about 2 weeks under conditions at $20-30{ }^{\circ} \mathrm{C}$ with a light and dark cycle of 14 and $10 \mathrm{~h}$, respectively. The $M$. oryzae isolates were grown on oatmeal agar plates in the dark for 7 days at $28{ }^{\circ} \mathrm{C}$ and then cultured under light for 5 days at room temperature for sporulation. Before inoculation, the cement tank was partially covered with a plastic membrane tent. Rice seedlings were spray-inoculated with spores at a concentration of $5 \times 10^{5}$ spores $\mathrm{mL}^{-1}$. After spraying, the cement tank was completely covered with the tent, and the inoculated seedlings were maintained under dark conditions for $24 \mathrm{~h}$. The tent was then removed from the tank, and the seedlings were grown in the greenhouse under high humidity for a further week for symptom evaluation.

\section{DNA extraction}

Leaf tissues from rice seedlings were collected for DNA extraction. About $100 \mathrm{mg}$ young leaf samples collected in Eppendorf tubes were frozen in liquid nitrogen, and were ground into fine powder with a TissueLyser (Qiagen, Haan, Germany). Rice genomic DNA was extracted from ground tissues by the CTAB (Cetyltrimethyl Ammonium Bromide) method as mainly described by Murray and Thompson (1980).

\section{DNA sequence analysis}

The genomic sequences of the Pi2/9 locus in the rice cultivars C101A51 (DQ352453), 75-1-127 (DQ285630), and Nipponbare (DQ454158), and the nucleotide sequence of the Piz- $t$ gene in the rice cultivar Toride-1 (DQ352040) were retrieved from the GenBank database of the National Center for Biotechnology Information (http://www.ncbi.nlm.nih.gov). Based on a preliminary multiple sequence comparison of DQ352453, DQ285630, DQ454158, and DQ352040, a primer pair P9-F/P9-R (Additional file 1: Table S1) was designed and utilized to amplify the allelic fragments corresponding to the promoter region of $\mathrm{Pi} 9$ and its alleles from C101A51, Toride-1, 75-1-127, Nipponbare, and 22 other rice cultivars (Fig. 1b). Similarly, a primer pair 2-LRR-F/ 2-LRR-R (Additional file 1: Table S1) was designed to amplify the allelic sequences bearing the polymorphisms for the first six amino-acid differences between Pi2 and Piz-t within the LRR motifs (Zhou et al. 2006). Primers 2-LRR-F/2-LRR-R were subsequently utilized in PCR amplification using genomic DNA templates of C101A51, Toride-1, 75-1-127, Nipponbare and 20 other cultivars (Fig. 2b). PCR products from primers P9-F/P9$\mathrm{R}$ and 2-LRR-F/2-LRR-R were sequenced using an ABI3730 XL automatic DNA sequencer. DNA sequences were aligned using Clustal Omega (Sievers et al. 2011).

\section{Development of allele-specific markers for Pi2 and Pi9}

Based on the multiple sequence alignment of 26 sequences of the promoter region of Pi9 and its alleles, primers 9-Pro-F/9-Pro- $\mathrm{R}$ were designed to develop a marker targeting the InDel polymorphisms among the Pi2/Pizt, Pi9, and non-Pi2/Piz-t/Pi9 alleles. PCR was performed under standard conditions with about $50 \mathrm{ng}$ of rice genomic DNA as template. The PCR products were separated in $10 \%$ polylacrylamide gels and then visualized by silver-staining. Based on the multiple sequence alignment of 24 sequences within the LRR coding region of the $\mathrm{Pi} 2$ and non-Pi2 alleles, a CAPS marker was designed to differentiate the Pi2 and nonPi2 alleles by using the dCAPS Finder 2.0 program (Neff et al. 2002) for the identification of appropriate restriction enzymes. PCR amplicons from primers 2-LRR-F/2LRR-R were digested with PstI or HinfI. Cleavage of PCR amplicons was performed at $37{ }^{\circ} \mathrm{C}$ for $4 \mathrm{~h}$ under the following conditions: $10 \mu \mathrm{L}$ of the PCR amplicon, 2 $\mathrm{uL} 10 \times$ buffer (TaKaRa, Dalian, China), and $1 \mathrm{uL}$ of the restriction enzymes PstI or HinfI (TaKaRa, Dalian, China) in a final volume of $20 \mu \mathrm{L}$. The enzyme-digested products were separated in $10 \%$ polylacrylamide gels and then visualized by silver-staining.

\section{Genotyping in diverse Chinese rice cultivars or breeding materials with allele-specific markers}

Genomic DNAs of the 434 Chinese rice cultivars or breeding materials were subjected to genotyping for the presence of Pi2 and Pi9 using the newly developed InDel marker Pi9-Pro and CAPS marker Pi2-LRR. In addition, two previously reported CAPS/dCAPS markers Pi2SNP and Pi9SNP (Hua et al. 2015) which targeted the coding region of Pi2 
and $P i 9$ respectively, were applied for genotyping. For genotyping using the Pi2SNP marker, PCR amplicons from primers Pi2SNP-F/Pi2SNP-R (Additional file 1: Table S1) were digested with Hinfl. And for genotyping using the Pi9SNP marker, PCR amplicons from primers Pi9SNP-F/ Pi9SNP-R (Additional file 1: Table S1) were digested with HindIII. The enzyme-digested products were separated electrophoretically in $10 \%$ polylacrylamide gels.

\section{Additional files}

Additional file 1: Table S1. Primers used in this study. (DOC $38 \mathrm{~kb}$ )

Additional file 2: Table S2. Rice cultivars and breeding materials assessed in this study. (DOC $51 \mathrm{~kb}$ )

\section{Abbreviations}

CAPS: cleaved amplified polymorphic sequence; CTAB: cetyltrimethyl ammonium bromide; dCAPS: derived cleaved amplified polymorphic sequence; InDel: insertion-deletion; LRR: leucine-rich repeat; MAS: markerassisted selection; NBS-LRR: nucleotide binding site-leucine-rich repeat; SNP: single nucleotide polymorphism; SSR: simple sequence repeat.

\section{Competing interests}

The authors declare that they have no competing interests.

\section{Authors' contributions}

DT carried out the experiments; ZC, ZC participated in inoculation analysis, allelism analysis, genotyping analysis; $Y Z$ participated in the design of the study; ZW and FW participated in inoculation analysis, genotyping analysis and in interpretation of data; SC was responsible for conception and planning of the study, interpretation of results and writing the manuscript. All authors read and approved the final manuscript.

\section{Acknowledgements}

We are grateful to Dr. Guo-Liang Wang, The Ohio State University, for kindly providing the rice lines C101A51, Toride-1 and 75-1-127, and the blast isolates PO6-6, KJ201, CHE86 and CHNOS, and to Dr. Daniel J. Ebbole for editing the manuscript. This project is supported by grants from National Natural Science Foundation of China (U1405212, U1305211) and grant from Natural Science Foundation of Fujian Province, China (2014 J07004).

\section{Author details}

${ }^{1}$ College of Crop Science, Fujian Agricultural and Forestry University, Fuzhou 350002, China. ${ }^{2}$ Biotechnology Research Institute, Fujian Academy of Agricultural Sciences, Fuzhou 350003, China. ${ }^{3}$ Fujian-Taiwan Joint Center for Ecological Control of Crop Pests, Fujian Agriculture and Forestry University, Fuzhou 350002, China.

Received: 5 January 2016 Accepted: 14 April 2016

Published online: 04 May 2016

\section{References}

Andersen JR, Lubberstedt T (2003) Functional markers in plants. Trends Plant Sci 8:554-560. doi:10.1016/.j.tplants.2003.09.010

Ashkani S, Rafii MY, Shabanimofrad M, Ghasemzadeh A, Ravanfar SA, Latif MA (2016) Molecular progress on the mapping and cloning of functional genes for blast disease in rice (Oryza sativa L.): current status and future considerations. Crit Rev Biotechnol 36:353-367. doi:10.3109/07388551.2014.961403

Chen Z, Zheng Y, Wu W, Zhao C (2004) Screening and application of an SSR marker closely linked to $\mathrm{Pi}-2(\mathrm{t})$, a gene resistant to rice blast (In Chinese with English summary). Mol Plant Breed 2:321-325. doi:10.3969/j.issn.1672-416X. 2004.03.003

Correa-Victoria F, Martinez C (2009) Breeding rice cultivars with durable blast resistance in colombia. In: Wang GL, Valent B (eds) Advances in genetics, genomics and control of rice blast disease. Springer, Netherlands, pp 375383. doi:10.1007/978-1-4020-9500-9_36
Costanzo S, Jia Y (2010) Sequence variation at the rice blast resistance gene Pi$\mathrm{km}$ locus: implications for the development of allele specific markers. Plant Sci 178:523-530. doi:10.1016/j.plantsci.2010.02.014

Dean RA, Talbot NJ, Ebbole DJ, Farman ML, Mitchell TK, Orbach MJ, Thon M, Kulkarni R, Xu JR, Pan H, Read ND, Lee YH, Carbone I, Brown D, Oh YY, Donofrio N, Jeong JS, Soanes DM, Djonovic S, Kolomiets E, Rehmeyer C, Li W, Harding M, Kim S, Lebrun MH, Bohnert H, Coughlan S, Butler J, Calvo S, Ma LJ, Nicol R, Purcell S, Nusbaum C, Galagan JE, Birren BW (2005) The genome sequence of the rice blast fungus Magnaporthe grisea. Nature 434:980-986. doi:10.1038/nature03449

Deng Y, Zhu X, Shen Y, He Z (2006) Genetic characterization and fine mapping of the blast resistance locus Pigm(t) tightly linked to Pi2 and Pi9 in a broad spectrum resistant Chinese variety. Theor Appl Genet 113:705-713. doi:10. 1007/s00122-006-0338-7

Ebbole DJ (2007) Magnaporthe as a model for understanding host-pathogen interactions. Annu Rev Phytopathol 45:437-456. doi:10.1146/annurev.phyto. 45.062806.094346

Fjellstrom R, McClung AM, Shank AR (2006) SSR markers closely linked to the Pi-z locus are useful for selection of blast resistance in a broad array of rice germplasm. Mol Breed 17:149-157. doi:10.1007/s11032-005-4735-4

Frisch M, Bohn M, Melchinge AE (1999) Minimum sample size and optimal positioning of flanking markers in marker-assisted backcrossing for transfer of a target gene. Crop Sci 39:967-975. doi:10.2135/cropsci1999. $0011183 \times 003900040003 x$

Fu C, Wu T, Liu W, Wang F, Li J, Zhu X, Huang H, Liu Z, Liao Y, Zhu M, Chen J, Huang $Y$ (2012) Genetic improvement of resistance to blast and bacterial blight of the elite maintainer line Rongfeng B in hybrid rice (Oryza sativa L.) by using marker-assisted selection. Afr J Biotechnol 11:13104-13124. doi:10.5897/AJB12.1465

Hayashi K, Yoshida H, Ashikawa I (2006) Development of PCR-based allele-specific and InDel marker sets for nine rice blast resistance genes. Theor Appl Genet 113:251-260. doi:10.1007/s00122-006-0290-6

Hayashi K, Yasuda N, Fujita Y, Koizumi S, Yoshida H (2010) Identification of the blast resistance gene Pit in rice cultivars using functional markers. Theor Appl Genet 121:1357-1367. doi:10.1007/s00122-010-1393-7

Hua L, Wang W, Chen S, Wang C, Zeng L, Yang J, Zhu X, Su J (2015) Development of specific DNA markers for detecting the rice blast resistance gene alleles Pi2/9/z-t (In Chinese with English summary). Chin J Rice Sci 29: 305-310. doi:10.3969/j.issn.1001-7216.2015.03.010

Jena KK, Mackill DJ (2008) Molecular markers and their use in marker-assisted selection in rice. Crop Sci 48:1266-1276. doi:10.2135/cropsci2008.02.0082

Jeung JU, Kim BR, Cho YC, Han SS, Moon HP, Lee YT, Jena KK (2007) A novel gene, Pi40(t), linked to the DNA markers derived from NBS-LRR motifs confers broad spectrum of blast resistance in rice. Theor Appl Genet 115:1163-1177. doi:10.1007/s00122-007-0642-x

Jia Y, Wang Z, Singh P (2002) Development of dominant rice blast Pi-ta resistance gene markers. Crop Sci 42:2145-2149. doi:10.2135/cropsci2002.2145

Jiang H, Feng Y, Bao L, Li X, Gao G, Zhang Q, Xiao J, Xu C, He Y (2012a) Improving blast resistance of Jin $23 \mathrm{~B}$ and its hybrid rice by marker-assisted gene pyramiding. Mol Breed 30:1679-1688. doi:10.1007/s11032-012-9751-6

Jiang N, Li Z, Wu J, Wang Y, Wu L, Wang S, Wang D, Wen T, Liang Y, Sun P, Liu J, Dai L, Wang Z, Wang C, Luo M, Liu X, Wang GL (2012b) Molecular mapping of the Pi2/9 allelic gene Pi2-2 conferring broad-spectrum resistance to Magnaporthe oryzae in the rice cultivar Jefferson. Rice 5:29. doi:10.1186/19398433-5-29

Jiang J, Mou T, Yu H, Zhou F (2015) Molecular breeding of thermo-sensitive genic male sterile (TGMS) lines of rice for blast resistance using Pi2 gene. Rice 8:11. doi:10.1186/s12284-015-0048-3

Khanna A, Sharma V, Ellur RK, Shikari AB, Gopala Krishnan S, Singh UD, Prakash G, Sharma TR, Rathour R, Variar M, Prashanthi SK, Nagarajan M, Vinod KK, Bhowmick PK, Singh NK, Prabhu KV, Singh BD, Singh AK (2015) Development and evaluation of near-isogenic lines for major blast resistance gene(s) in Basmati rice. Theor Appl Genet 128:1243-1259. doi:10.1007/s00122-015-2502-4

Liu G, Lu G, Zeng L, Wang GL (2002) Two broad-spectrum blast resistance genes, Pig(t) and Pi2(t), are physically linked on rice chromosome 6. Mol Genet Genomics 267:472-480. doi:10.1007/s00438-002-0677-2

Liu W, Wang F, Jin S-J, Zhu X-Y, Li J-H, Liu Z-R, Liao Y-L, Zhu M-S, Huang H-J, Fu F-H, Liu Y-B (2008) Improvement of rice blast resistance in TGMS line by pyramiding of Pi-1 and $\mathrm{Pi}-2$ through molecular marker-assisted selection (In Chinese with English summary). Acta Agron Sinica 34:1128-1136. doi:10.3724/SP.J.1006.2008.01128 
Liu W, Liu J, Triplett L, Leach JE, Wang GL (2014) Novel insights into rice innate immunity against bacterial and fungal pathogens. Annu Rev Phytopathol 52: 213-241. doi:10.1146/annurev-phyto-102313-045926

Liu J, Li J, Qu J, Yan S (2015) Development of genome-wide insertion and deletion polymorphism markers from next-generation sequencing data in rice. Rice 8:27. doi:10.1186/s12284-015-0063-4

Luo Y, Yin Z (2013) Marker-assisted breeding of Thai fragrance rice for semi-dwarf phenotype, submergence tolerance and disease resistance to rice blast and bacterial blight. Mol Breed 32:709-721. doi:10.1007/s11032-013-9904-2

McCouch SR, Teytelman L, Xu Y, Lobos KB, Clare K, Walton M, Fu B, Maghirang R, Li Z, Xing Y (2002) Development and mapping of 2240 new SSR markers for rice (Oryza sativa L.). DNA Res 9:199-207. doi:10.1093/dnares/9.6.199

Murray MG, Thompson WF (1980) Rapid isolation of high molecular weight plant DNA. Nucleic Acids Res 8:4321-4325. doi:10.1093/nar/8.19.4321

Neff MM, Turk E, Kalishman M (2002) Web-based primer design for single nucleotide polymorphism analysis. Trends Genet 18:613-615. doi:10.1016/ S0168-9525(02)02820-2

Qu S, Liu G, Zhou B, Bellizzi M, Zeng L, Dai L, Han B, Wang GL (2006) The broadspectrum blast resistance gene Pig encodes a nucleotide-binding siteleucine-rich repeat protein and is a member of a multigene family in rice. Genetics 172:1901-1914. doi:10.1534/genetics.105.044891

Ramkumar G, Srinivasarao K, Madhan Mohan K, Sudarshan I, Sivaranjani AKP, Gopalakrishna K, Neeraja CN, Balachandran SM, Sundaram RM, Prasad MS, Shobha Rani N, Rama Prasad AM, Viraktamath BC, Madhav MS (2011) Development and validation of functional marker targeting an InDel in the major rice blast disease resistance gene Pi54 (Pik $\left.{ }^{h}\right)$. Mol Breed 27:129-135. doi:10.1007/s11032-010-9538-6

Ramkumar G, Prahalada GD, Hechanova SL, Vinarao R, Jena KK (2015) Development and validation of SNP-based functional codominant markers for two major disease resistance genes in rice (O. sativa L.). Mol Breed 35:129. doi:10.1007/s11032-015-0323-4

Sievers F, Wilm A, Dineen DG, Gibson TJ, Karplus K, Li W, Lopez R, McWilliam H, Remmert M, Soding J, Thompson JD, Higgins DG (2011) Fast, scalable generation of high-quality protein multiple sequence alignments using Clustal Omega. Mol Syst Biol 7:539. doi:10.1038/msb.2011.75

Suh JP, Roh JH, Cho YC, Han SS, Kim YG, Jena KK (2009) The Pi40 gene for durable resistance to rice blast and molecular analysis of Pi40-advanced backcross breeding lines. Phytopathology 99:243-250. doi:10.1094/PHYTO-99-3-0243

Tsunematsu H, Yanoria MJT, Ebron LA, Hayashi N, Ando I, Kato H, Imbe T, Khush GS (2000) Development of monogenic lines for rice blast resistance. Breed Sci 50:229-234. doi:10.1270/jsbbs.50.229

Wen S, Gao B (2011) Introgressing blast resistant gene Pi-9(t) into elite rice restorer Luhui17 by marker-assisted selection. Rice Genomics Genet 2:31-36. doi:10.5376/rgg.2011.02.0004

Wu JL, Fan YY, Li DB, Zheng KL, Leung H, Zhuang JY (2005) Genetic control of rice blast resistance in the durably resistant cultivar Gumei 2 against multiple isolates. Theor Appl Genet 111:50-56. doi:10.1007/s00122-005-1971-2

Yin D, Xia M, Li J, Wan B, Zha Z, Du X, Qi H (2011) Development of STS markers linked to rice blast resistance gene Pig in marker-assisted selection breeding (In Chinese with English summary). Chin J Rice Sci 25:25-30. doi:10.3969/j. issn.1001-7216.2011.01.004

Zeigler RS, Tohme J, Nelson R, Levy M, Correa-Victoria FJ (1994) Lineage exclusion: a proposal for linking blast population analysis to resistance breeding. In: Zeigler RS, Leong SA, Teng PS (eds) Rice blast disease. CAB International, Wallingford, pp 267-292

Zhou B, Qu S, Liu G, Dolan M, Sakai H, Lu G, Bellizzi M, Wang GL (2006) The eight amino-acid differences within three leucine-rich repeats between $\mathrm{Pi2}$ and $\mathrm{Piz}$ $t$ resistance proteins determine the resistance specificity to Magnaporthe grisea. Mol Plant Microbe Interact 19:1216-1228. doi:10.1094/MPMI-19-1216

Zhu X, Chen S, Yang J, Zhou S, Zeng L, Han J, Su J, Wang L, Pan Q (2012) The identification of Pi50(t), a new member of the rice blast resistance Pi2/Pi9 multigene family. Theor Appl Genet 124:1295-1304. doi:10.1007/s00122-012$1787-9$

\section{Submit your manuscript to a SpringerOpen ${ }^{\circ}$ journal and benefit from:}

- Convenient online submission

- Rigorous peer review

- Immediate publication on acceptance

- Open access: articles freely available online

- High visibility within the field

- Retaining the copyright to your article

Submit your next manuscript at $\gg$ springeropen.com 